\title{
Protée
}

\section{Marie Calumet dans la littérature québécoise. Pour une sémiotique de l'histoire littéraire}

\section{Fernand Roy}

Volume 27, numéro 2, 1999

La réception

URI : https://id.erudit.org/iderudit/030558ar

DOI : https://doi.org/10.7202/030558ar

Aller au sommaire du numéro

\section{Éditeur(s)}

Département des arts et lettres - Université du Québec à Chicoutimi

ISSN

0300-3523 (imprimé)

1708-2307 (numérique)

Découvrir la revue

Citer cet article

Roy, F. (1999). Marie Calumet dans la littérature québécoise. Pour une sémiotique de l'histoire littéraire. Protée, 27(2), 45-56.

https://doi.org/10.7202/030558ar
Résumé de l'article

L'hypothèse à étayer était la suivante : les discours qui lient dans un corpus les textes déjà reçus comme littéraires par la critique immédiate génèrent la notion d'histoire comme interprétant apte à rendre compte, au moyen de l'écriture, du devenir d'une collectivité. Un examen systématique de la réception particulière qui a été réservée au roman Marie Calumet - de sa mise à l'Index en 1904 à sa consécration après 1960, en passant par sa réédition en 1946 - a permis de montrer 1) que les histoires littéraires n'ont pas inventé la « valeur » du texte : en l'avalisant uniquement après 1960, elles ont tout bonnement reconduit les perceptions de la critique immédiate ; 2) que le texte incitait déjà à se servir de l'écriture pour gérer le quotidien ; et 3) que, entre 1904 et 1970, ce n'est pas le roman qui a changé mais bien la notion d'histoire l'anecdote appelait un renouvellement de la notion. 


\section{MARIE CALUMET D AN S LA LITTÉRATU RE Q U ÉBÉCO ISE POUR UNE SÉM IO TIQ UE DE L'HISTO IRE LITTÉRAIRE ${ }^{1}$}

FERNAND ROY

En 1904, la critique fut veule; en 1946, elle fut stupide. Marie Calumet est un livre important de notre littérature, beaucoup plus que ceux de Laberge dont c'est la mode de dire du bien dans les revues universitaires.

(Jacques Ferron, 1970a: 75)

\section{PROBLÉMATIQUE ET HYPOTHĖSE DE TRAVAIL}

S'il convient de savoir que le roman Marie Calumet a été reconnu par l'institution littéraire québécoise quelque cinquante ans après sa publication, soit au moment où l'on a commencé à parler de spécificité québécoise en littérature, il convient par ailleurs et tout autant de ne pas méconnaître qu'en 1904 c'est uniquement la critique canadienne-française qui avait été «veule»: en 1907, dans ses Nouvelles études de la littérature canadienne française (sic), Charles ab der Halden (p. 317-318) parlait du jeune romancier Rodolphe Girard comme d'un écrivain intéressant et curieux. Force est donc de relativiser un tant soit peu le propos de Jacques Ferron, et de faire d'entrée de jeu l'hypothèse que le jugement des historiens d'une littérature donnée évolue en fonction de la définition qu'ils donnent à la notion d'histoire qu'ils privilégient ${ }^{2}$. Aussi, analyser l'intégration progressive de Marie Calumet à l'histoire de la littérature québécoise implique à la fois de prendre en compte ce que la vision actuelle - québécoise - de la littérature doit à Marie Calumet et de chercher à identifier la fonction de l'histoire dans l'intégration de Marie Calumet au corpus littéraire québécois. À partir du fait que la vision de l'histoire privilégiée influe directement sur les textes constitutifs du corpus d'une littérature donnée, serait-il possible, en «renversant la vapeur», d'étayer l'idée que la notion d'histoire a comme préalable l'existence de textes déjà reçus comme littéraires?

S'il est impossible d'imaginer une histoire littéraire sans imaginer un certain nombre de textes lui préexistant, on ne saurait par contre nier que la spécificité - ou non - du littéraire ne va pas sans qu'il y ait littérature. Sans prétendre répondre ici dans l'absolu à la question «la poule ou l'œuf?», je me permettrai de 
préciser un peu le fondement théorique - socio-sémiolinguistique - de ma réflexion. Je pose qu'une (structure de) signification est toujours déjà l'effet, sur un matériau -sonore ou écrit, en l'occurrence ici en cause - donné, d'un processus d'interaction (possiblement fictif) entre deux interlocuteurs, disons $\mathrm{X}$ et $\mathrm{Y}$; qu'un consensus fonde l'existence du signe linguistique fait que cette interaction n'est, à proprement parler, verbale que la seconde fois que $\mathrm{X}$ - ou Y - émet les mêmes sons - en présence de l'autre qui réactualise le consensus établi. La nécessité du signe peut être entendue concrètement: après avoir anticipé une motivation aux sons émis par $\mathrm{X}$, après avoir en quelque sorte fait l'hypothèse - abduction que ces sons constituaient un signal eu égard au lieu de $\mathrm{X}, \mathrm{Y}$ actualise un signal eu égard à son lieu à lui ; la mise en rapport des deux signaux fait du second un interprétant recevable du premier - interprétant dont $\mathrm{X}$ confirme finalement la pertinence. On se doit cependant de remarquer que le processus d'interaction constitutif d'une langue fonde en quelque sorte symboliquement l'interdépendance matérielle de X et de Y, en occultant éventuellement la différence entre le lieu de X et celui de Y. Il me paraît nécessaire de ne pas confondre

l'interdépendance structurelle et la différence qui est, elle, conjoncturelle: la première ne va pas sans la seconde, mais l'intérêt de la distinction tient justement à ce qu'elle permet de comprendre que la seconde ne conduit pas obligatoirement à la première.

La perspective socio-historique propose d'analyser les discours en lisant la distance inévitable entre les interlocuteurs en termes de rapports sociaux de production. Pour rendre compte de la fortune de Marie Calumet depuis 1904, elle parlera d'une intervention dans le processus de production d'une langue "commune" à une collectivité et mettra en évidence que le roman met en forme une lutte pour la possession du capital symbolique, lutte qui, en dernière instance, est elle-même surdéterminée par la lutte des classes. Le roman apparaitra alors comme créateur d'illusion, justement en ce qu'il raconte une histoire où l'hégémonie culturelle du clergé est battue en brèche au profit d'une vision plus «libérale» de l'écriture: le point de vue «laïque» du «journaliste» Rodolphe Girard allait à long terme triompher du point de vue «religieux» du curé, mais cette libération s'avérerait illusoire puisqu'elle impliquait en dernière instance un asservissement du travail d'écriture du journaliste aux intérêts de la bourgeoisie libérale - le mariage de l'homme engagé du curé avec Marie Calumet a lieu au presbytère, mais il ne va pas sans un contrat de mariage - plein de clauses économiques «symboliquement» rédigé par le notaire sur la table de travail du curé.

La perspective sociosémiotique me semble un peu différente qui, elle, toujours pour rendre compte de la «fortune littéraire» de Marie Calumet, voudra plutôt décrire en termes de processus discursif les diverses péripéties qui ont mené à l'intégration du roman au corpus littéraire national, sans lier directement ces diverses étapes à l' "asservissement» de l'institution aux intérêts de la bourgeoisie libérale: elle s'appliquera à montrer que la prise en compte du roman par l'histoire littéraire constitue une étape décisive dans la mise en place du discours constitutif de l'institution littéraire québécoise. Cela donnera éventuellement aux tenants de la perspective sociohistorique l'impression que pour un sociosémiologue, la littérature québécoise est un phénomène qui n'a rien d'historique; alors que cela revient simplement à essayer de penser la structure de l'institution avant de l'analyser à partir de ce qui la surdétermine. À noter que pour les fins de mon propos, cette perspective sociosémiotique est à distinguer de la problématique dite de la «réception": je veux justement suggérer ici que les théories de la réception auraient avantage à distinguer entre la réception immédiate des textes par la critique et le traitement que font subir aux textes et à leur réception critique les histoires littéraires. Pour une raison bien simple: les phénomènes langagiers sont irréductibles à une dyade "action/ réaction». Ils sont - deviennent- langagiers parce qu'ils résultent d'un processus interactif qu'il serait simpliste de prétendre décrire de l'intérieur en termes de «question-réponse». À mon sens, la théorie 
«réception-réponse» à un «texte-question » ne gagne en rien à être retournée dans un utopique hors-temps en termes de «trouver-la-question» à laquelle le «texte-seveut-une-réponse». Ce qui lui manque, à la théorie «question/réponse», c'est d'arriver à assumer que le résultat est acquis par la mise en place dans le temps d'une sanction de la «parole-réponse» par la «questionen-tant-que-désormais-abolie».

Il me paraîtrait réducteur de prétendre choisir entre les deux perspectives, la socio-historique et la sociosémiotique. J'explorerai plutôt l'idée - l'espoir!que les deux projets sont non pas contradictoires mais bien à articuler dans un rapport de complémentarité. Compte tenu de ce qu'une hypothèse de travail vaut par ce qu'elle permet d'inscrire dans la logique d'un savoir, je fais celle que la visée sémiotique présuppose - au sens sémiotique, donc de façon univoque - la visée sociologique ${ }^{3}$.

Pour arriver à intégrer l'histoire littéraire dans le processus de reconnaissance de Marie Calumet par l'institution littéraire québécoise, il me paraît utile, dans un premier temps, de faire appel à une problématique sémiotique d'analyse de discours. Cependant, le projet sémiotique ne saurait inclure l'étude du champ littéraire en tant que lieu d'une lutte pour la possession du capital symbolique; aussi, une fois que l'on aura réussi à penser l'histoire littéraire en termes de composante du discours constitutif de l'institution littéraire, il sera normal d'en venir au projet sociologique d'étude de cette institution. Ce projet aura à prendre en compte le fait qu'il s'agit d'une institution essentiellement - et non pas seulement conjoncturellement - liée à la pratique du langage dans les sociétés d'écriture, et à la [re]production d'une langue nationale: on ne saurait en réduire la spécificité à une question de lutte dans le champ du capital symbolique.

À partir de ces positions théoriques, en analysant le processus d'intégration lente du roman Marie Calumet à la littérature québécoise, j'espère illustrer l'utilité de penser que la constitution d'une histoire littéraire initie la phase de légitimation - par des études savantes - des textes reçus comme littéraires au moment de leur publication. Il me paraît utile de définir l'amorce de cette phase de légitimation en termes de performance sémionarrative: on ne saurait définir adéquatement le concept sans l'envisager dans une perspective discursive, sans y voir une transformation visant à légitimer - par l'interprétant «histoire»- le texte qui lors de sa publication était donné comme problématique - du fait justement qu'il n'avait pas encore été reçu comme littéraire. L'intérêt de l'opération est de taille: il y va de la mise en évidence que l'histoire littéraire rendrait possible une lecture historique de l'évolution sociale d'une collectivité, une fois que certains textes se donnant comme représentatifs de cette évolution ont été reçus comme littéraires par la critique immédiate. Alors que la publication du texte constituerait un nouveau - donc problématique - contrat de lecture, l'histoire littéraire avaliserait socialement ce contrat à la lumière de la réception immédiate. Cette phase de transformation est à distinguer clairement de la phase de réception critique immédiate qui, elle, consiste plutôt à faire l'hypothèse de la littérarité même, en affirmant l'autonomie du texte.

Essentiellement, je propose que l'histoire littéraire constitue en corpus les textes déjà reçus comme littéraires : ce serait un discours intégrateur qui générerait l'histoire comme «interprétant» valable pour rendre compte de l'évolution d'une collectivité.

J'insiste: mon hypothèse consiste à dire que c'est la catégorie "histoire» qui résulte du "corpus constitué»; alors que la validation de la valeur littéraire même des textes intégrés ne peut être dégagée qu'après coup, pour cautionner la coloration donnée à la catégorie "histoire». En d'autres termes: Marie Calumet n'a pas changé pour la peine entre sa parution en 1904 et la fin, imminente, du second millénaire; ce qui a changé au cours du siècle, c'est la notion d'histoire.

Je développerai mon propos en deux temps. D'abord je m'appliquerai à montrer que le roman Marie Calumet a dû être reconnu comme littéraire par la critique immédiate avant d'être intégré dans le corpus littéraire québécois; je le ferai en soulignant que cela s'est produit au moment où la société 
québécoise se donnait une vision moderne - libéralede l'histoire. Puis j'essayerai de montrer que c'est une vision de l'histoire - et non pas une vision du littéraire!- que Marie Calumet cautionne depuis 1960.

\section{MARIE CALUMET}

Un roman reconnu littéraire 50 ans après sa parution Le premier point à étayer est que l'histoire littéraire n'a pas inventé l'étiquette littéraire qu'elle a reconnue à Marie Calumet. L'histoire littéraire n'a pas défini la spécificité interne - la littérarité- de Marie Calumet; une fois le roman reçu positivement par la critique immédiate, elle s'est simplement appliquée à l'intégrer dans le corpus littéraire - en s'en servant comme d'un anti-sujet "préhistorique», ce qui sera le second point de ma démarche.

En 1904, Rodolphe Girard exerçait le métier de journaliste à La Presse et il avait déjà, aux yeux de ses pairs intellectuels, le statut d'homme de lettres en herbe: il avait publié un roman de jeunesse, Florence, en 1900. En 1907, il en publia un troisième, Rédemption; et un quatrième, L'Algonquine, parut en 1910, dans le journal Le Temps. Pourtant, et même si dès 1903 La Presse avait déjà publié une entrevue de Girard parlant du «roman de mœurs» qu'il préparait, aucune histoire littéraire écrite au Canada et consacrée spécifiquement à la littérature canadiennefrançaise n'a parlé de Rodolphe Girard avant 19604. La plus haute instance de la critique institutionnelle, l'archevêché de Montréal, avait immédiatement condamné Marie Calumet à l'oubli, en le mettant à l'Index: il présentait un «danger de perversion morale, esthétique et littéraire» (La Semaine religieuse, 1904: 87).

En ignorant l'œuvre de Girard, Camille Roy - et à sa suite Albert Durand et Samuel Baillargeon - s'est tout simplement montré respectueux de la position prise par l'archevêque de Montréal. À mon avis, ce respect des «événements» était une condition de la reconnaissance de Camille Roy comme historien de la littérature. Dans son «Avant-propos» aux Origines littéraires, à peine cinq ans après la condamnation de Marie Calumet, Camille Roy parlait du souci de "conserver intact le patrimoine sacré de notre langue française» et ajoutait que «si imparfaites que soient les œuvres dont nous parlons, elles sont éminemment représentatives de la vie d'une époque [et ...] des documents que nous ne pouvons pas ignorer" (Roy, 1909: 7-8). Quelque vingt ans plus tard, son silence au sujet de tous les romans de Girard signifiait donc que ce dernier n'était toujours pas un homme de lettres important: Marie Calumet n'était pas parmi les "documents que nous ne pouvons pas ignorer»; ainsi en avait décidé la collectivité par le biais de ses critiques immédiats les plus patentés. On pourra évidemment souligner que le Nihil obstat qui apparaît dans tous les livres publiés par Camille Roy explique en partie cet état de fait: l'œuvre de Girard ne présentait pas d'intérêt "pour le patrimoine sacré de la langue française». Cela marque bien que l'objectif de Camille Roy était tout de même décalé par rapport à celui de la réception critique immédiate: il s'agissait d'inscrire les textes reçus positivement par la critique dans un discours constitutif du corpus officiel, en l'occurrence défini «patrimoine sacré de la langue française». On ne peut pas reprocher à Camille Roy d'avoir pris acte que le roman de Girard menaçait le caractère sacré de ce patrimoine: il faisait son métier d'historien, en conformité avec la coloration de la notion d'histoire-signe qui prévalait alors.

Pour la réédition du roman en 1946, Girard n'a pas pris de chance: il a "expurgé» son texte, c'est-àdire, entre autres, en l'amputant, à mon avis malencontreusement, d'une bonne partie du dernier chapitre; et le roman a été précédé d'une généreuse introduction de son ami Albert Laberge, où il était question de «chef-d'œuvre» (Laberge, 1946) ${ }^{5}$. Cette seconde fois, Marie Calumet a été reçu de façon moins farouchement négative. La Revue populaire a pourtant été la seule à aller ouvertement dans le sens de Laberge et à parler d'un «livre extraordinaire» attendu par «le public» «depuis quarante ans» (La Revue populaire, 1947 : 22). Dans Lectures, on a prudemment convenu qu'il s'agissait d'un récit "vivant» et «alerte», qui «tourn[ait] parfois au grotesque» mais qui méritait tout de même d'être pris en compte par 
l'institution littéraire (Laplante, 1947: 121] ${ }^{6}$. De son côté, La Presse a reproduit la critique mitigée de Culture, où Romain Legaré avait parlé d'un «roman populaire et non proprement littéraire ou artistique» (Legaré, 1947 : 363] ${ }^{7}$. Cependant, la prestigieuse Revue de l'Université Laval, fidèle à son célèbre historien de la littérature, s'est montrée carrément plus sévère: À peine un roman [...] le tout est désobligeant et n'a pas le moindre accent de vérité canadienne [...] grasse caricature dégô̂tante [qui] aurait pu rester dans l'oubli.

(repris dans La Revue populaire, 1947: 794)

Mais la réception immédiate dans son ensemble a été suffisamment positive pour que Gérard Tougas soit tenu - objectivité d'historien oblige - de consentir une place à Rodolphe Girard, dès 1960. Je dis "consentir" car il s'agit d'une place plus à l'ombre qu'au soleil. À toutes fins pratiques, Tougas prit acte du petit scandale soulevé en 1904, question de donner du poids à la vision libérale et laïcisante de l'histoire qu'il proposait de substituer à celle, plus conservatrice et plus cléricale, de Camille Roy. Le propos de Tougas mérite une brève analyse.

Après avoir très rapidement qualifié Marie Calumet de «roman aussi innocent que fade» et de "Clochemerle affadi [ayant] causé, lors de sa publication, un petit scandale qui lui a permis de survivre», au moment de conclure son survol du roman québécois d'avant 1940, Tougas n'en prenait pas moins appui sur Rodolphe Girard:

Le roman canadien est libre. Son asservissement à quelques grands thèmes, liés à la survivance française, n'a, du reste, jamais existé que dans son esprit. S'il est vrai que certains romanciers, tels Rodolphe Girard et Jean-Charles Harvey, ont encouru la vindicte [...], qui oserait affirmer, en plaçant le débat sur le seul plan des idées et du bon goût, que la littérature canadienne, du fait de l'intervention des bien-pensants, s'en est trouvée dangereusement appauvrie?

(Tougas, 1967: 155)

J'attirerai d'abord l'attention sur le manque d'originalité du propos de Tougas. Dans la revue Lectures, on avait déjà dit que le roman de Girard n'avait « rien de l'irrespect flagrant et de l'irréligion affichée dans les textes de l'auteur de Clochemerle» (Laplante, 1947: 121). Le "Clochemerle affadi» retenu illustre bien que le discours de l'historien constitue essentiellement une lecture-synthèse de la réception critique récente du roman. Mais l'essentiel est l'idée de la fin de l'asservissement à des grands thèmes. Cette interprétation permet: 1) de rendre compte du scandale causé par le roman de Girard; 2) de respecter le succès moyen de la réédition tout en justifiant le silence momentané de l'histoire de Camille Roy: les curés ont eu bien tort de s'en prendre à un petit roman aussi innocent, mais ils n'en avaient pas moins des idées et du goût; et 3) de suggérer une cohérence à l'ensemble mis en place: il y a eu évolution lente et progressive vers la liberté. Ce qui caractérise en somme le propos de Tougas et le distingue de celui de Roy, c'est le souci de constituer le patrimoine de la langue française, sans en faire quelque chose de sacré. À ses yeux, Marie Calumet demeure une ouvre bien imparfaite; mais il répète après Camille Roy que «si imparfaites que soient les œuvres dont nous parlons [ce sont] des documents que nous ne pouvons pas ignorer». La différence entre les deux points de vue tient simplement à ce que la suite discursive de Tougas avance l'idée de la libération progressive du roman des thèmes imposés par le clergé: la vraie littérature est libre, elle n'a pas d'autre finalité qu'elle-même. Pour rendre vraisemblable cette idée de libération progressive de la littérature, Tougas la fait naître dans le temps: il donne Marie Calumet comme preuve de l'étroitesse d'esprit du clergé. Ce qui lui "permet" évidemment de ne pas remarquer qu'il se contente de remplacer le paradigme "patrimoine sacré» par un autre paradigme tout aussi utopique.

Marie Calumet est donc un beau cas pour commencer à circonscrire le rôle de l'histoire littéraire dans la constitution d'un corpus littéraire. D'une part, à la suite de la condamnation de 1904, le silence d'un Camille Roy au sujet de Girard montre que l'historien de la littérature travaille, comme les autres historiens, sur des documents et que ses documents sont les textes qui ont été élevés au rang de "valeurs» 
par la critique immédiate. D'autre part, l'entrée de Marie Calumet dans le corpus, en 1960, fait suite à la re-publication du roman en 1946: Tougas a adapté la position d'abord adoptée par l'historien Camille Roy à la critique dont a été l'objet le texte lors de sa réédition ${ }^{8}$. Il ne lui est pas venu à l'esprit de revoir la notion de «valeur» même: il s'est au contraire appliqué à souligner la sûreté de goût de son prédécesseur. Ce qui donne à penser que ce qui change d'une histoire littéraire à l'autre c'est en effet l'interprétant "histoire», et non pas la valeur littéraire même.

D'UN «ROMAN DE MEEURS» (en 1903)

À «UNE SIMPLE SUITE DE TABLEAUX» (en 1980)

Comme Marie Calumet n'avait pas fait l'objet d'un consensus «institutionnel» positif au début du siècle, le contrat - entre l'éditeur et les lecteurs - dans sa totalité a dû être relancé en 1946. L'œuvre devait, cette fois, passer la rampe, mais sans que la critique immédiate soit unanime. Cela explique peut-être le parti qu'allait prendre Réédition-Québec en 1969, en revenant à la version "originale» de $19044^{9}$. Il y a bien sûr aussi que, tout de suite après 1960, il a été convenu, dans le milieu universitaire laïcisant et participant de la révolution tranquille, qu'il existait effectivement une littérature québécoise ${ }^{10}$. Dans un livre-synthèse portant sur Le Roman canadien-français, l'historien Paul Wyczynski devait conclure que Marie Calumet "disloque le cadre régi du roman canadien, trop exclusivement porté, jusque-là, à idéaliser la vie» (1964: 17) et constitue le premier jalon d'une littérature populaire, une sorte de clin d'œil: «une grosse farce efficace, bien menée et accessible».

En faisant de Marie Calumet le premier des quatre romans les plus marquants des premières décennies du siècle, Wyczynski révisait le travail commencé par Tougas. "Cité» dans une optique d'affirmation de la spécificité de la littérature d'ici, Marie Calumet allait bientôt faire date dans l'histoire du roman canadienfrançais. Et l'histoire littéraire continue encore actuellement à le considérer comme une "date», c'està-dire comme un texte qui vaut par son contenu plus que par sa valeur littéraire: c'est devenu l'histoire de la servante qui hésite à jeter la sainte pisse de monseigneur...

Je pense avoir contribué ailleurs à démontrer que Marie Calumet n'est pas une «simple suite de tableaux» mais bien un roman de facture on ne peut plus régulière ${ }^{11}$. Ce que je veux m'appliquer à préciser maintenant, c'est qu'en définitive l'attribution d'un statut historique à Marie Calumet fonde la notion d'histoire socioculturelle que véhicule encore aujourd'hui le discours qui prétend décrire la vie littéraire au Québec. Mon argumentation tendra à montrer qu'en méconnaissant l'organisation d'ensemble de ce récit, et en occultant de ce fait sa valeur, les historiens de la littérature québécoise continuent de jauger la vie littéraire à l'aune des catégories servant à analyser la vie sociale. En faisant de la libération religieuse le point de départ de l'écriture littéraire, ils continuent de mythifier le savoir historique et d'idéaliser la fonction sociale de l'écriture.

Le paradoxe est que, ce faisant, les historiens de la littérature répètent on ne peut plus fidèlement le discours que tenait déjà Rodolphe Girard en 1903. Après avoir annoncé la publication prochaine d'une étude de mœurs sur la fameuse Marie Calumet de la chanson populaire (La Presse, 1903: 10), La Presse publiait, également en 1903, le résultat d'une entrevue avec l'"homme de lettres" Rodolphe Girard. Celui-ci y expliquait que son roman serait une «étude de mœurs faite dans des milieux délaissés [...] par nos littérateurs au Canada» (Charlebois, 1986: 75). Il affirmait avoir fait «un travail à la fois éthologique et philologique» et prévenait que son roman pourrait peut-être passer pour «un livre de patois». «Je ne songerai pas à m'en défendre autrement, ajoutait-il, adepte que je suis de cette école réaliste qui met la vérité historique au-dessus de la convention littéraire» (ibid., p. 76). Girard était donc conscient d'aller contre les canons littéraires de son temps, notamment en ce qui concerne l'utilisation du parler populaire dans un texte littéraire. Ce qui est moins évident, par contre, c'est pourquoi l'histoire littéraire allait, même après 1960, traduire en termes 
d'imperfection formelle le danger de "perversion" littéraire vu dans le roman lors de sa parution.

D'évidence, on touche là une corde sensible: jusqu'à quel point une institution liée à la reproduction d'une langue dont la norme est écrite est-elle tenue d'illustrer cette norme? À sa manière, Girard avait résolu le problème en précisant qu'il mettait la vérité historique au-dessus de la convention littéraire. Ce qui impliquait que la convention littéraire se devait d'être au service de l'histoire-processus.

En 1969, il y a eu la réimpression en fac-similé de l'édition de 1904, par Réédition-Québec; en 1973, Luc Lacoursière a assuré la reprise de l'édition de 1946 dans la prestigieuse collection du Nénuphar; et, depuis, d'abord en 1979 puis en 1990, le roman a été réédité dans la collection «Bibliothèque québécoise» ${ }^{12}$. Que les reprises de 1979 et de 1990 n'aient guère retenu l'attention de la critique s'entend facilement: le roman était, dans l'immédiat, devenu «événement» dans la "préhistoire» du roman québécois; et l'éditeur assure simplement la présence du livre dans les librairies, pour répondre à la demande du marché scolaire ${ }^{13}$.

Ce n'est pas seulement par la magie de l'édition prestigieuse de 1973 que «Le galeux devient un respectable personnage " ${ }^{14}$. Constituée par un universitaire de renom, la collection du Nénuphar était plutôt de l'ordre d'une sanction définitive de l'appartenance du roman au corpus littéraire québécois; toutes proportions gardées, elle était de l'ordre d'une édition critique. Dès 1970, en effet, la réception critique immédiate a revu et corrigé le jugement mitigé antérieur dont Tougas avait proposé une synthèse libérale. À cette occasion, une nouvelle ligne de pensée s'est dessinée, qui est depuis reprise par les histoires littéraires ${ }^{15}$. La réception de cette troisième édition du roman allait être tout aussi déterminante que la seconde pour les historiens de la littérature: Marie Calumet «n'est ni grossier, ni immoral, ni impie" (Robin, 1970: 18); ce n'est cependant pas un très bon roman, plutôt «un événement plus sociologique que littéraire ${ }^{16}$, mais il était temps que les historiens de la littérature le reconnaissent. Jacques Ferron avait donné le ton en écrivant qu'il s'agissait d'un «chef-d'œuvre d'un genre qui nous est propre: la monographie de paroisse» (Ferron, 1970a: 75) et dont l'interdiction allait faire de l'écrivain québécois «un intellectuel colonisé» par la France (idem, 1970b: 52).

Ferron n'a évidemment pas été le seul à valoriser la couleur locale du roman. Jean Blouin faisait parallèlement la manchette de "Perspectives», le supplément culturel hebdomadaire du quotidien Le Soleil, en y rappelant en quatre pages «Le scandale de Marie Calumet " (1970). Cette tendance à relire le passé avait été précédée, tant dans $\mathrm{La}$ Presse que dans $\mathrm{La}$ Patrie et le Dimanche-Matin, d'invitations à prendre «quelques heures de bonne humeur» (Martel, 1970: 32), question de savoir qui était Marie Calumet ( $\mathrm{a}$ Patrie, 1970: 17). Jacques Vigneault, dans Québec-Presse, se montrait plus incisif en proposant une interprétation psychosociale qui dépassait, tout en mythifiant le travail d'écriture, le choix de la «vérité» historique qu'avait annoncé Girard:

L'analyse un peu serrée [...] révèle un message qui devrait glacer

d'horreur le lecteur pénétrant [...], un arrêt à la phase anale [...]. L'univers de l'œeuvre [...] entièrement dominé par la crainte et la tentation d'une régression à cette phase de la vie, n'est qu'une lutte entre propreté et saleté, dressage et instinct. On ne saurait expliquer autrement cette obsession de la propreté [...].

Marie Calumet de Rodolphe Girard est une autre preuve de

l'abrutissement auquel confinait une éducation qui refoulait

l'activité sexuelle, réprimait l'imagination [...].

(Vigneault, 1970: 14a)

En dépit de tous ces jugements critiques positifs, l'histoire littéraire allait continuer de considérer, à la suite de Tougas, le petit roman comme bien imparfait mais digne de parvenir à la postérité en tant que «date».

Il est intéressant de voir que l'impact de la réception critique des rééditions de 1969 et de 1973 sur les propos des historiens n'est pas allé sans un décalage ${ }^{17}$. Je citerai d'abord des histoires publiées à la fin des années soixante, c'est-à-dire datant de la réception critique des années cinquante: 
Ce roman, d'ailleurs médiocre et qui nous paraît aujourd'hui anodin, a pourtant soulevé en son temps des protestations indignées.

(Bessette et alii, 1968: 368)

Les premières tentatives de roman psychologique remontent au début du XX e siècle, avec des romanciers comme Rodolphe Girard (Florence, 1900, Marie Calumet, 1904, Rédemption, 1906) [...] mais rien ne vaut véritablement d'être signalé avant que ne surgisse [...], dans les années 1930, JeanCharles Harvey.

(Grandpré, 1968: 109)

[...] farce cléricalo-villageoise de Rodolphe Girard, dont la langue grasse et l'odeur excrémentielle firent scandale.

(Mailhot, 1974: 35)

[...] farce que certains passages facétieusement stercoraires permettent de qualifier de rabelaisienne [...] n'est qu'une très innocente satire des moeurs cléricales de la campagne.

(Dionne, 1984: 92)

[...] cet ensemble de tableaux qu'on hésite parfois à appeler roman, constitue une ouvre qui vaut surtout par son côté humoristique et sa finesse de ton.

(Lafortune, 1985: 27)

Alors qu'en 1968, le roman continuait d'être une œuvre "médiocre» ayant soulevé de la poussière lors de sa parution, voilà qu'au tournant des années quatre-vingt, il est devenu une farce implicitement satirique, un «ensemble de tableaux" dont la forme laisse à désirer, mais qui vaut par son côté humoristique et sa finesse de ton. Au-delà des changements de mentalité, on pourrait, somme toute, s'attendre à ce que les historiens actuels rééditent l'avis de Gérard Tougas au sujet du jugement critique porté par le clergé sur Marie Calumet: les clercs manquaient d'ouverture d'esprit - et de sens de l'humour-, mais ils savaient reconnaître les textes valables. Les historiens de la littérature n'inventent pas la «valeur» qu'ils reconnaissent aux textes!

Je retiendrai ici pour fin d'une analyse un peu plus élaborée le jugement d'André Vanasse dans le Dictionnaire des œuvres littéraires du Québec (Lemire, 1980), jugement qui me paraît bien résumer le sentiment des historiens des trente dernières années, soit que Marie Calumet est une suite de tableaux - par opposition à un récit solidement construit - mais une «suite de tableaux, amusante parfois" et qui, surtout, aura eu le mérite de poser la "bonne question":

Marie Calumet est subversif non pas tant à cause des raisons

évoquées mais parce qu'il met en parallèle certains passages de la

Bible avec la vie du presbytère. En lisant les extraits du

Cantique des cantiques, que l'auteur donne à lire dans son roman, on ne peut s'empêcher de penser que la sexualité est fortement réprimée au pays du Québec. Qui a raison? La Bible ou ses représentants? Telle est la question non formulée mais clairement inscrite dans le récit. (Ibid., p.675)

$\mathrm{Du}$ «danger de perversion morale, esthétique et littéraire» qui avait motivé l'exclusion du texte du "patrimoine sacré» au début du siècle, à la «simple suite de tableaux" mais "subversive» qui justifie que le roman soit considéré comme une date en 1980, on peut se demander si la différence a à voir avec le littéraire ou avec la morale. Un indice de réponse se trouve dans le fait que Vanasse semble tenir moins à la qualité du texte qu'à la question qu'il y décrypte.

Le postulat d'une "question non formulée mais clairement inscrite» dans une simple «suite de tableaux" a un double effet. Subrepticement, il permet d'imaginer que le lieu d'où est formulée ladite question est objectif, historiquement parlant - elle fonde un discours qui va dans le sens de l'histoireprocessus encore valorisée aujourd'hui. Et, dans le même mouvement, la question se justifie comme lecture interprétative, puisque le document est posé comme n'ayant pas de cohérence interne - «simple suite de tableaux". De façon assez amusante, somme toute, un «roman» - on ne peut plus explicitement organisé - continue, soixante-quinze ans après sa parution, à être perçu comme déficient littérairement, mais il est devenu important dans l'histoire du Québec. Se pourrait-il que même après Le Cabochon et Les Belles-sours on reconduise encore et toujours, sur le dos des «milieux traditionnellement délaissés» par l'institution littéraire, une tradition qui se refuse à reconnaître la valeur littéraire de Marie Calumet? Se pourrait-il que l'idée de «subversion» y serve 
simplement, et un peu facilement en l'occurrence, d'exact envers de l'idée de "patrimoine sacré», sans que ne soit clairement faite la distinction entre la reproduction de la vie en général et celle de la langue au moyen de l'écriture en particulier?

En posant que le roman de Girard oppose tacitement le Cantique des cantiques et la vie au presbytère, Vanasse se sert hors contexte de «certains tableaux» qu'il lit manifestement dans une perspective autre que celle qui est construite dans le texte de Girard. Quant à savoir quel écrit le texte de Girard oppose explicitement au Cantique des cantiques, Vanasse donne ni plus ni moins sa langue au chat: on n'a pas à se poser la question, puisqu'il s'agit d'une simple "suite de tableaux" sans véritable cohésion. Quand on la pose, on a la surprise de découvrir que, structurellement, le texte oppose au langage poétique difficile du Cantique des cantiques le langage administratif tout aussi difficile du contrat de mariage entre Marie et Narcisse: le notaire fait un spécial et se rend rédiger le contrat au presbytère, très précisément sur la table de travail du curé, où la jeune nièce avait trouvé la Bible ouverte au Cantique des cantiques - ce qui lui avait appris que l'Écriture ne refusait pas la sexualité. La difficulté de la lecture de Vanasse est que Marie Calumet ne questionne pas la vie au presbytère, mais bien plutôt l'usage restreint de l'Écriture qui était traditionnellement fait au presbytère, avant la découverte par la jeune nièce du Cantique des cantiques. À un usage de l'Écriture réservé aux curés et centré sur les relations entre les humains et l'au-delà, le texte oppose un usage lié à la spontanéité et au bon sens des petites gens, spontanéité et bon sens qui les amènent à trouver dans l'Écriture une inspiration pour résoudre des problèmes concernant le quotidien. Et, en cela, l'ironie de Girard aidant, c'est le curé Flavel même qui pave la voie à ses paroissiens: malgré son manque de sens esthétique ${ }^{18}$, le hasard de la vie l'amène à découvrir que la lecture peut aussi être utile dans la gestion du quotidien, contrairement à ce qu'il avait appris au séminaire et qui faisait qu'il se croyait, par sa formation intellectuelle, spécialiste des seuls rapports entre les humains et l'éternel. L'anecdote du roman ouvre d'ailleurs sur une discussion où la «vision» du curé Flavel est remise en cause par le propos d'un collègue, le curé d'une paroisse plus prospère - «plus évoluée».

L'analyse de l'organisation narrative du roman montre que l'acte héroïque qui vaut à Narcisse le cœur de la servante du curé est inspiré de ses souvenirs scolaires - l'Histoire sainte. Qui plus est, cet acte héroïque décisif est précédé dans la diégèse par un autre acte héroïque: le curé Flavel sauve un homme de la noyade, au lieu de s'en tenir à sa mission divine, en se contentant de donner les derniers sacrements à un mourant. Cet acte ${ }^{19}$ héroïque se produit à la suite de la lecture de poèmes «inspirés» de Lamartine - lecture qui lui tient lieu de formation esthétique. La nuance est d'importance: l'anecdote valorise l'Écriture en la disant également utile dans diverses situations quotidiennes, à la condition d'avoir un minimum de sens esthétique; alors que Vanasse se contente de proposer que l'Écriture est en contradiction avec la vie au presbytère, sans prendre en compte que le texte suggère qu'il s'agit tout simplement d'un manque dans la formation du curé. En mettant trop exclusivement l'accent sur la sexualité, Vanasse s'empêche de pouvoir souligner clairement que Girard prêchait somme toute déjà pour son clocher laïcisant à lui, en tant que journaliste bien de son temps et qui subordonnait les canons esthétiques à la "vérité» historique. À cause de cette petite confusion, le texte de Vanasse laisse une impression curieuse: le lecteur a le sentiment qu'il a été écrit pour faire croire à l'histoire-processus, mais qu'il n'y arrive qu'en déniant que l'écriture littéraire actualise justement la possibilité de faire cette histoire.

Le propos de Vanasse n'est pas défavorable au romancier Girard. Bien au contraire, ce propos en fait un lecteur plus ou moins génial et plus ou moins en avance sur son temps. Toutes proportions gardées, Bourdieu (1992) fait à l'occasion la même chose de Flaubert, soit en en faisant quelqu'un qui met en représentation avec une singulière lucidité la lutte dans le champ culturel à une époque donnée. Ce qui revient à continuer de mythifier l'écriture: le résultat 
est qu'une proposition de langue commune imaginée dans l'écriture devient la preuve de la pertinence «scientifique» de la notion d'histoire. La chose est singulièrement évidente dans le texte de Vanasse: il y est implicitement suggéré que le petit roman de Girard a le grand mérite de permettre à certains intellectuels québécois des années soixante-dix de penser qu'ils ont su poser la bonne question. En retraçant dans une simple «suite de tableaux" cette bonne question, on trouve, et littéralement, dans l'écriture le sens de l'écriture même, soit la subversion de l'idéologie dominante. Mieux, d'une part on occulte l'histoiresigne et, d'autre part, de cette occultation, on tire le principe de vérité de l'histoire-processus.

En résumé: en 1904 Girard proposait, avec Marie Calumet, une vision de l'écriture littéraire qui ne pourrait recevoir une sanction positive que de la part d'une institution littéraire laïcisée. En suggérant d'infléchir les conventions littéraires pour aller dans le sens de l'évolution économique et sociale dont il participait, Girard proposait non pas de renouveler la notion de littéraire, mais bien de valoriser la notion d'histoire-processus. On peut prétendre, je pense, que la portée pratique de sa proposition lui échappait un peu et qu'il n'y pouvait rien: la seule manière de faire des propositions justes serait de s'assurer que la conjoncture est stable; ce qui était loin d'être le cas au pays du Québec au tournant du 20e siècle. Aussi, il ne faut peut-être pas s'étonner outre mesure de ce que la réponse de l'histoire littéraire à sa proposition déforme cette dernière jusqu'à en faire un indice précurseur de la modernité québécoise. Ce qui retient plutôt mon intérêt, c'est qu'à sa façon Girard faisait déjà une lecture tout aussi déformante sans doute de la Marie Calumet du 19e siècle célébrée par la chanson populaire. Comme quoi le littéraire participe toujours déjà, comme par définition, de la reproduction du langage.

\section{SÉMIOTIQUE ET HISTOIRE LITTÉRAIRE}

Du projet de Camille Roy au DOLQ (Dictionnaire des ceuvres littéraires du Québec), l'histoire littéraire fait bien son travail: elle construit une vision de l'histoire, en intégrant dans un corpus cohérent les textes reconnus comme littéraires par la critique immédiate. D'une part, elle réserve à Marie Calumet un statut mitigé quant à sa valeur littéraire; mais d'autre part elle présente, dans le temps, une vision différente de la société. Il me semble donc possible d'affirmer que le changement relatif des canons littéraires, d'une époque à l'autre, relève tout autant de la définition d'histoire que privilégient les historiens que de la valeur littéraire proprement dite.

Le défi serait évidemment d'arriver à démontrer que Camille Roy pensait au fond la littérature exactement comme Rodolphe Girard: on arriverait alors à donner raison aux sociologues de la littérature qui prétendent que la lutte pour la domination du champ culturel surdétermine le quotidien des institutions littéraires. Il convient sans doute aussi de comprendre que le classicisme a marqué les débuts de la littérature française en imposant une norme; et que c'est justement parce que la littérature française, comme institution, est née classique qu'on peut encore aujourd'hui imaginer que la valeur littéraire relève de l'intemporel, ne renvoie pas à l'écriture et à une problématique de reproduction du langage. Comment en effet ne pas évoquer ici la thèse de Cassirer selon laquelle il y aurait un dieu à l'origine de chacun des mots: pour en sortir, il faut sans doute imaginer une pré-littérature, comme c'est le cas de Marie Calumet, en littérature québécoise.

Est-ce à dire qu'une sémiotique de l'histoire littéraire proposant de voir dans l'histoire-processus l'interprétant qui fonde la littérature confinerait à l'invention d'un interprétant autre, qui serait apte à fonder une sorte d'histoire scientifique de la littérature? Je ne pense pas. Il me paraît plus juste de penser qu'il est possible de décrire scientifiquement le littéraire, et qu'il est possible aussi de comprendre que faire l'histoire de l'écriture est la condition sans laquelle cette description scientifique ne saurait exister. Si, en effet, on ne pouvait pas imaginer qu'il est possible de dire l'origine d'une littérature et de retracer son évolution, on pourrait difficilement 
prétendre à une description scientifique du phénomène.

En plaçant le projet d'une science du littéraire du côté de la sanction d'une démarche discursive inscrite dans le processus de reproduction du langage, on contribuerait, je pense, à rendre l'histoire de la vie littéraire encore plus exacte sociologiquement parlant: autrement, faute d'une définition exhaustive et falsifiable du littéraire, on risquerait de se dire encore longtemps que le littéraire c'est de la littérature. N'estce pas ce que faisait déjà, en son temps, Monseigneur Bourget en mettant Marie Calumet à l'Index au nom d'une vérité éternelle?

\section{N O TES}

1. Le travail de recherche ayant conduit à la rédaction du présent article a été subventionné par le CRSH et le FCAR. Le projet dans son ensemble portait sur la fonction des figures de l'écrit dans le roman québécois d'avant 1960; la première responsable était Louise Milot. Il m'aurait par ailleurs été impossible d' écrire le présent article si je n'avais pas eu accès à la documentation du DOLQ, disponible au CRELIQ de l'Université Laval.

2. À moins, bien évidemment, que l'on ne prenne le parti de se faire croire que l'histoire est une forme de savoir qui n'est pas elle-même liée à l'existence de l'écriture, comme le fait Renée Balibar (1991) dans l'exemple de ses travaux par ailleurs éminemment intéressants. 3. Ce qui n'implique en rien une négation du principe de surdétermination économique: il y a lieu de distinguer entre analyse de discours et analyse sociale.

4. En 1950, Auguste Viatte avait allégué qu'après 1870, pour un temps, la prose littéraire avait été le fait de journalistes et d'historiens et tout juste noté en passant que Rodolphe Girard avait publié dans une «langue affreuse des romans goguenards et assez bassement naturalistes" (1954: 152).

5. À noter également que cette préface était suivie d'un extrait d'un texte de Bernard Muddiman paru en 1911 dans le Queen's Quaterly où il était rappelé que Charles ad der Halden décrivait Girard comme «le jeune romancier canadien le plus intéressant et le plus curieux...» et dit de Marie Calumet qu'il s'agissait du «first great novel Canada has produced in either French or English" (Laberge, 1946: 16).

6. La mention «Pour adultes» apparaissait toutefois entre le titre du livre et le commentaire critique.

7. L'article a été reproduit dans La Presse du 18 octobre 1947.

8. D'où la possibilité inverse qu'une œuvre d'abord intégrée à un corpus tombe temporairement au moins hors du corpus officiel.

9. Comme Rodolphe Girard a lui-même collaboré à l'édition de 1946, il convient de considérer qu'il s'agit de l'édition définitive.

10. Un exemple n'a jamais fait mal à personne. Au début des années soixante, j'ai eu comme professeurs de littérature québécoise trois personnes dont deux ont par la suite fait leur marque en littérature québécoise, Jacques Blais, Maurice Lemire et Yves de Margerie. Le premier m'étonnait beaucoup en soutenant que selon lui Saint-Denys Garneau était le premier grand écrivain québécois, alors que le troisième me disait que la littérature québécoise commençait réellement avec l'École littéraire de Montréal. Maurice Lemire, pour sa part, nous suggérait plutôt qu'il fallait savoir reconnaître que la spécificité québécoise tenait à ce que notre première littérature a été une littérature du terroir. Comme j'avais pour ma part acquis dès le collège classique l'habitude de lire Nelligan et même Crémazie et Aubert de Gaspé, je dois avouer que je trouvais le point de vue de Jacques Blais plutôt surprenant. Il a publié une édition critique des textes de Louis Fréchette.

11. Voir mon introduction dans la dernière édition de la Bibliothèque québécoise (Fides, 1990) et l'article "L'inscription de l'écriture dans Marie Calumet » que j'ai cosigné avec L. Milot et F. Ouellet (1990: 8095).

12. À noter que cette édition intègre en annexe la version de 1904 du dernier chapitre, le plus amputé par l'édition définitive de 1946. 13. Il n'est guère, en milieu universitaire québécois, de cours de panorama de la littérature québécoise où les étudiants ne sont pas tenus de lire Marie Calumet.

14. L'expression est de J.-Y. Théberge dont le compte rendu paru dans Le Canada français en octobre 1973 a été repris dans Vient de paraître, vol.4, no 1 (mars 1974).

15. Il n'est évidemment pas dit que le présent est absolument garant de l'avenir; on peut fort bien imaginer que l'histoire littéraire de demain fasse de Marie Calumet le classique québécois dont parlait Jacques Ferron en 1970.

16. L'expression est tirée du compte rendu paru dans Le Livre canadien en 1974.

17. Ne serait-ce qu'à cause du temps de préparation puis des délais de publication d'une histoire littéraire.

18. Manque de sens esthétique qu'il convient manifestement de relier à la formation tronquée qui fut la sienne: il n'avait pas réussi les dernières années de son cours classique, mais on l'avait accepté au séminaire étant donné qu'il avait la vocation.

19. On pourrait dire "lapsus", puisque le mourant pour lequel le curé a été appelé est tout simplement laissé pour compte.

\section{RÉFÉREN CES BIBLIO g RAPHIQ U ES}

BALIBAR, R. [1991] : Histoire de la littérature française, Paris, P.U.F, coll. "Que sais-je?»;

[1974]: Les Français fictifs, Paris, Hachette. Bessette, G., L. GesLin, et C. PAREnt [1968]: Histoire de la littérature canadienne-française, Montréal, CEC.

BlouIN, J. [1970]: «Notre premier roman humoristique ne fit pas rire du tout», Le Soleil ("Perspectives», vol. 12, no 13), vol. 86, n 73, 20-24. 
Bourdieu, P. [1992]: Les Règles de l'art, Paris, Seuil.

CHARLEBOIs-DischaUer, M. [1986] : Rodolphe Girard (1879-1956): sa

vie, son œuvre, Montréal, Fides.

DiONNE, R. [1984]: Le Québécois et sa littérature, Sherbrooke, Naaman. FERron, J. [1970a]: «Marie Calumet », Le Petit Journal, vol.44, n²2, 75 ;

[1970b]: «Dessaules réhabilité», Magazine Maclean (mai), 51-52. GRANDPRÉ, P. de [1968]: Histoire de la littérature française du Québec, tome 2, Montréal, Beauchemin.

HALDEN, C. ab der [1907]: Nouvelles études de littérature canadienne française [sic], Paris, F. R. de Rudeval.

L. B. [1947] : "Compte rendu ", dans "Notes bibliographiques", La

Revue de l'Université Laval (mai), 794.

Laberge, A. [1946]: «Préface» de R. Girard, Marie Calumet, Montréal,

Brousseau, 9-13.

LaFORTUNE, M., [1985]: Notre roman, Laval, Mondia.

LAPLANTE, R. [1947]: «Compte rendu», Lectures, vol.2, n², 121.

La Patrie [1970]: «Pour savoir qui elle était, il faut lire Marie Calumet », vol. $91, \mathrm{n}^{\circ} 12,27$.

La Presse [1903]: "Grignotades", vol. 19, nº174 (30 mai), 10.

La Revue populaire [1947]: "Vient de paraittre», (avril), 22.

La Semaine religieuse de Montréal [1904]: «Un mauvais livre», vol. 18, $\mathrm{n}^{\circ} 6$.

LEGARÉ, R. [1947]: «Livres canadiens», Culture, vol. 8 (septembre), 363. Le Livre canadien [1974]: «Compte rendu», n²58 (septembre).
LEMIRE, M. (dir.) [1980]: Dictionnaire des aeuvres littéraires du Québec, tome 2, Montréal, Fides.

MAILHOT, L. [1974]: La Littérature québécoise, Paris, P.U.F., coll. «Quesais-je?».

MARTEL, R. [1970]: «Chronique Littérature», La Presse, vol. 86, n 73 , 32.

Milot, L., F. Ouellet et F. ROY [1990]: «L'inscription de l'écriture dans Marie Calumet ", Voix et Images, vol. 16, n 1, 80-95.

RoBIN, E. [1970]: «La paille et le grain», L'Information médicale et paramédicale, vol. 22, nº 14 .

RoY, C. [1909]: Nos Origines littéraires, Québec, Imprimerie de l'Action sociale.

RoY, F. [1990]: «Marie Calumet et l'institution littéraire», Introduction à Marie Calumet, Montréal, Fides, 7-20.

THÉBERGE, J.Y. [1973]: «Marie Calumet a bien marié l'engagé du curé [...]", Le Canada français, vol. 114, n²3, 80.

TOUGAS, G. [1967]: Histoire de la littérature canadienne-française, Québec et Paris, P.U.L. et P.U.F.

VIATTE, A. [1954]: Histoire de la littérature de l'Amérique française des origines à 1950, Québec, P.U.L.

Vigneault, J. [1970]: «Marie Calumet, un roman bien sale », QuébecPresse, vol.2, n $15,14 \mathrm{a}$.

WYCZYNSKI, P. [1964]: «Panorama du roman canadien-français", Le Roman canadien-français (Archives des lettres canadiennes-françaises, tome 3), Montréal, Fides, 11-35. 